\title{
Chemoprevention of lung cancers: lessons from CARET, the beta-carotene and retinol efficacy trial, and prospects for the future
}

\author{
Gilbert S. Omenn
}

The objective of this paper was to review the strategies for lung cancer chemoprevention. A retrospective assessment of the major findings from the most informative lung cancer chemoprevention clinical trials [alpha-tocopherol (vitamin $E)$, beta-carotene trial and $\beta$-carotene and retinol efficacy trial] was employed. Both trials and many others showed no benefit from what was once the prime candidate for lung cancer chemoprevention, $\beta$-carotene. Furthermore, both trials found that $\beta$-carotene, alone or in combination with vitamin $E$ or retinyl palmitate, increased the incidence of lung cancers and the total and cardiovascular mortality rates. In conclusion, design, conduct, documentation, relationships with participants, and preparedness for unexpected findings are all important for chemoprevention research. Trials are necessary to test inferences from observational epidemiology and animal models. Multiple

\section{Introduction}

Cancer prevention strategies originate from our understanding of the causes of various cancers (Greenwald, 2002). As shown in Table 1 , they may be grouped into four categories: (a) reducing high-risk behaviors; (b) controlling chemical, physical, and microbial carcinogenic agents; (c) chemoprevention with hormones, micronutrients, or pharmaceuticals; and (d) screening and treating preneoplastic lesions (Omenn, 2004a).

It is customary to describe preventive interventions in relation to the target populations. Primary prevention aims at the general population of healthy individuals, including subgroups with notable risk factors (high-risk populations). Secondary prevention focuses on alreadyscreened individuals, usually with premalignant lesions at particular sites, now including individuals at high risk for lung cancer with suspicious lesions after spiral computed tomography screening of the chest. Tertiary prevention refers to interventions in cancer survivors to prevent second primaries, recurrences, and/or complications after the presumed cure of their initial cancers.

A crucial concept in planning interventions is the recognition of the crucial influence of heterogeneity of underlying carcinogenic mechanisms, diagnostic features, and responsiveness to therapeutic and preventive interventions. Heterogeneity leads to subpopulation analyses and stratification for interventions, as well as recognition classes of promising agents are available for evaluation and for eventual randomized trials. European Journal of Cancer Prevention 16:184-191 (c) 2007 Lippincott Williams \& Wilkins.

European Journal of Cancer Prevention 2007, 16:184-191

Keywords: alpha-tocopherol (vitamin E), beta-carotene trial, $\beta$-carotene, $\beta$-carotene and retinol efficacy trial, chemoprevention, cyclooxygenase and lipoxygenase inhibitors, Israel, retinoids

University of Michigan, Ann Arbor, Michigan, USA

Correspondence and requests for reprints to Gilbert S. Omenn, MD, PhD, University of Michigan, Ann Arbor, MI 48109-0656, USA

Tel: +1 734763 7583; fax: +1 734647 8148; e-mail: gomenn@umich.edu

Received 25 August 2005 Accepted 28 September 2005

of variable pharmaco-genetic influences on biotransformation of the drug or on target-site susceptibility to its desired or adverse effects. Heterogeneity requires us to speak of 'cancers' in the plural. The emergence of new signaling pathways, receptors, and mRNA and protein expression patterns assists us in understanding the basis for heterogeneity and in identifying and validating molecular targets for interventions.

\section{Chemoprevention of lung cancers}

Lung cancers are the number 1 cause of cancer deaths in the United States and increasing in incidence and mortality around the world (Jemal et al., 2004). No mystery exists about the cause of most of the cases: $85 \%$ of cases in men and $70 \%$ in women occur in current or former cigarette smokers. Among the rest, second-hand smoke, radon, asbestos, and a few occupational chemical exposures account for most cases (IARC, 2004). Some evidence exists of genes predisposing to risk of lung cancers (Keith and Miller, 2005). Development of molecular biomarkers for lung cancers is a high priority for the US National Cancer Institute Early Detection Research Network, especially with the National Lung Cancer Screening Trial in 50000 high-risk individuals well launched using spiral computed tomography. Obtaining a biopsy of lung lesions is not a trivial procedure, so molecular biomarkers that could be used to evaluate individuals with suspicious lesions on imaging could be promptly put to good use. 
Table 1 Cancer prevention strategies flow from causes

- Behavioral: reducing high-risk behaviors Smoking cessation and smoking prevention Assuring healthy weight versus overweight Healthy diet - fruits and vegetables Avoiding unprotected sex, unprotected suntan

- Controlling chemical, physical, microbial agents that cause cancers; immunizing against exposures

- Chemoprevention

Hormones (tamoxifen first approved) Micronutrients (vitamins and minerals)

Chemopreventive pharmaceuticals (celecoxib)

- Screening and treating preneoplastic lesions

In the United States, there are 172000 new cases and 157000 deaths per year from lung cancers (Jemal et al., 2004). Despite tremendous efforts to diagnose the cancer earlier and to develop potent chemotherapy, 5-year survival from the time of diagnosis is still only $15 \%$, essentially the same as 40 years ago (when I graduated from medical school). The vast majority of the 'cures' (5-year survivors) remains those who were 'caught' in time for the surgeon to remove the tumor. Even among stage 1 patients, with $48 \%$ 5-year survival overall, molecular profiling shows marked heterogeneity for subgroups with very good versus much worse survival curves (Beer et al., 2002).

The situation in Israel is rather similar, on the basis of the data published from the Middle East Cancer Consortium (Freedman et al., 2003), updated at the Carmel and Dead Sea meetings of May 2004 in posters from the Israel National Cancer Registry. Incidence rates are lower than in the United States, reflecting lower prevalence rates of cigarette smoking. Among Jewish men, the incidence at 30 per 100000 has risen at $0.2 \%$ per year for 30 years, so that lung cancers are tied for number 3 with bladder cancer, after prostate and colorectal cancers. Among Jewish women, the incidence is 10 per 100000 , rising at $0.7 \%$ per year and ranking approximately fourth (with several other cancer sites) after breast (85), colorectal (30), and non-Hodgkin's lymphoma (12). Among Arab men in Israel, lung cancer incidence is 25 per 100000 , ranking number 1 in incidence, whereas the incidence in Arab women is much less than 10 per 100000 , ranking below the top five cancers.

Given the scale of recruitment and cost in terms of money and time of conducting large-scale, doublemasked, randomized clinical preventive trials, investigators and sponsors realize that there must be a strong scientific rationale for testing chemopreventive candidate agents for efficacy and safety in humans (Peto et al., 1981; Greenwald, 2002; Omenn, 2004b). The rationale emerges from a combination of presumed mechanisms of action (including potential toxicity) and of results from animal models, in-vitro model systems, epidemiologic associa- tions, and human intermediate endpoint trials and phase I/II safety and dose-ranging studies.

In the early 1980s, evidence of lower incidence of epithelial cancers in association with higher dietary intake of fruits and vegetables or measured blood levels of $\beta$-carotene or total carotenoids put the spotlight on antioxidants, and particularly $\beta$-carotene, as a chemopreventive candidate agent (Peto et al., 1981). Meanwhile, experimental laboratory evidence had generated much interest in retinoids (Sporn et al., 1976). Major trials (see below) were launched to test $\beta$-carotene, alone or in combination with vitamin $\mathrm{E}$ or vitamin A (Omenn, 1998). Antioxidants were hypothesized to reduce the peroxidation of surface features of cancer cells and more broadly counteract reactive oxygen species. Retinoids were proposed to have a complementary favorable action, namely inducing differentiation of epithelial cells, instead of proliferation, through action on RAR and RXR receptors in the nucleus. Most animal and in-vitro studies have been conducted with retinoids, as $\beta$-carotene is peculiarly insoluble in the aqueous phase of blood plasma, circulating instead within particles, mostly high-density lipoprotein-lipoprotein particles, and rats and mice are notoriously poor absorbers of $\beta$-carotene and other carotenoids from the intestine. As early as 1967 Saffiotti showed reduction in tumors with retinyl palmitate (Saffiotti et al., 1967). A key experiment (McCormick et al., 1980) later showed that the reduction in tumor incidence could be achieved with administration well after the exposure to the responsible carcinogen (7,12-dimethylbenz[a]anthracene) in the rat mammary system; this pattern of treatment clearly was relevant to the need to intervene in humans well after their exposures to cigarette smoking and other carcinogens (such as asbestos) began or even ended.

\section{Alpha-tocopherol (vitamin E), beta-carotene trial and $\beta$-carotene and retinol efficacy trial}

Table 2 identifies the main $\beta$-carotene cancer prevention trials (Omenn, 1998). The largest trials for chemoprevention of lung cancers in high-risk populations were ATBC, the alpha-tocopherol (vitamin E), beta-carotene trial in 29133 male smokers in Finland (ATBC, 1994), and CARET, the $\beta$-carotene and retinol efficacy trial in 14254

\section{Table 2 Beta-carotene trials}

- Alpha-tocopherol, beta-carotene trial (ATBC)
- Beta-carotene and retinol efficacy trial (CARET)
- Physicians' health study (Aspirin/Beta-Carotene)
- Skinen's health study (Beta-Carotene/ASA/vit E)
- Retinoic acid, beta-carotene, and 13-cis retinoic acid trials, head and neck,
- $\quad$ Surrodigestive (M.D. Anderson)
- Texas; France)
- Vitamins and minerals supplementation (Linxian trials)


Fig. 1

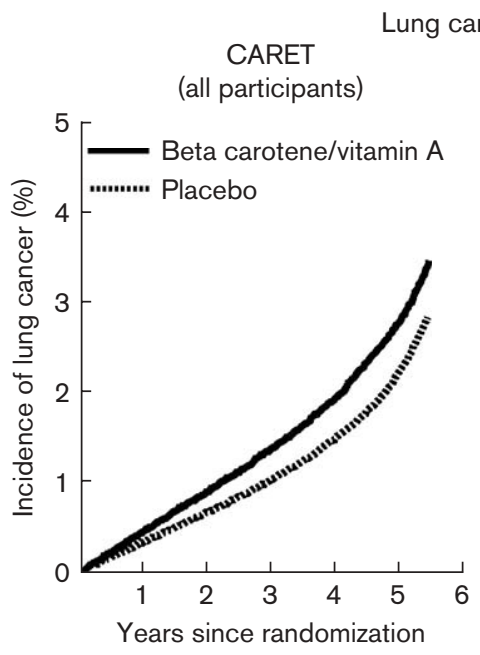

ATBC

(all participants)

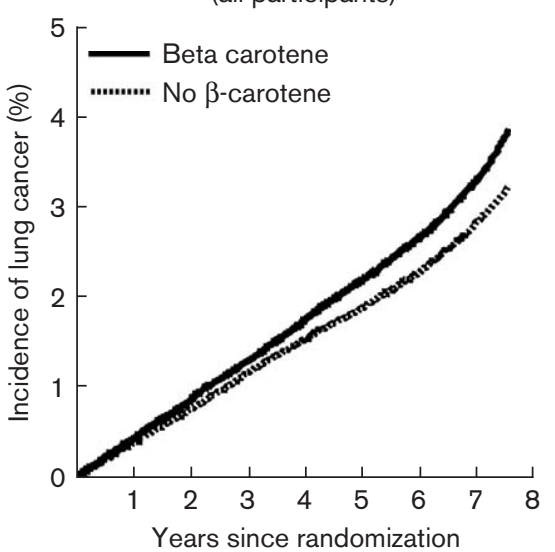

Lung cancer incidence in the alpha-tocopherol (vitamin E), beta-carotene trial (ATBC) and in the $\beta$-carotene and retinol efficacy trial (CARET), comparing active intervention with $\beta$-carotene (ATBC) or with a combination of $\beta$-carotene and retinyl palmitate (CARAT) versus placebo.

Fig. 2

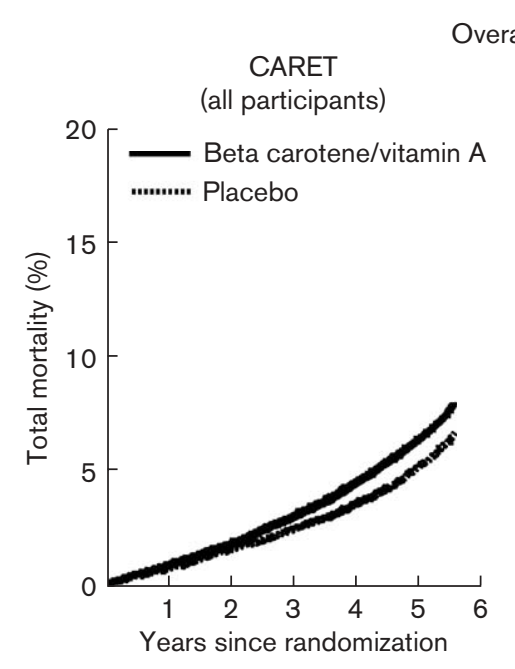

Overall deaths

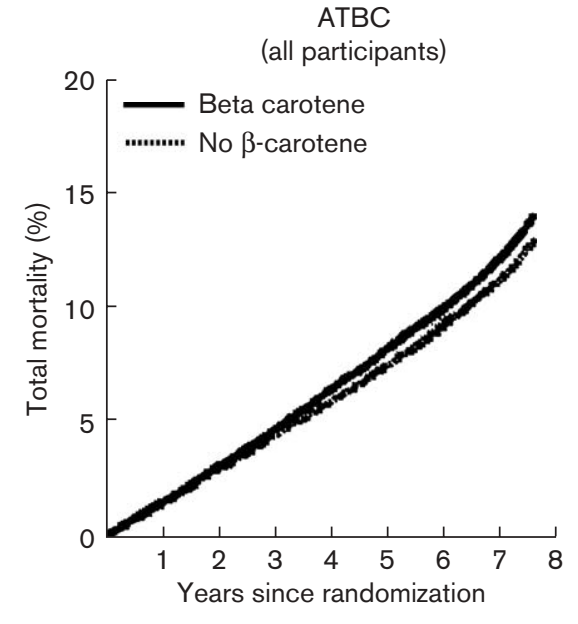

Mortality rates in the alpha-tocopherol (vitamin E), beta-carotene trial (ATBC) and in the $\beta$-carotene and retinol efficacy trial (CARET).

male and female current and former heavy smokers and 4060 male smokers and former smokers significantly exposed to asbestos in various occupations (Omenn et al., 1996a, b).

As is well-known with large-scale randomized trials, an enormous amount of work and tremendous cooperation from the trial participants yield results that can usually be published after many years in a single publication. Figure 1 shows the lung cancer incidence rates and Fig. 2 shows the mortality rates from ATBC and CARET. The results were stunningly similar: excess lung cancer incidence and excess mortality in the active vitamin treatment arm, compared with the placebo arm of each trial. ATBC reported $18 \%$ increased lung cancer incidence and $8 \%$ increased total mortality, and CARET reported $28 \%$ increased lung cancer incidence and $18 \%$ increased total mortality (ATBC, 1994; Omenn et al., 1996a, b). The data are summarized, 
Table 3 (a) ATBC trial in Finland and (b) beta-carotene and retinol efficacy trial (CARET) in US

(a) ATBC trial in Finland

- 29133 male smokers (age 50-69 years; $5+$ cig/day)

- Mean 36 pack-years

- $20 \mathrm{mg} \beta$-carotene $+50 \mathrm{IU}$ vit $\mathrm{E} \times 6.5$ years

- $10 \times$ and $5 \times$ median daily dietary intake

- Funded by $\mathrm{NCl}$; registries supported in Finland

- 874 lung cancer cases and 3570 deaths ascertained

- Good coordination with CARET

(b) Beta-carotene and retinol efficacy trial (CARET) in US

- 14254 heavy smokers (current/former, male \& female)

- 4060 men exposed occupationally to asbestos

- Beta-carotene $30 \mathrm{mg} / \mathrm{day}+$ retinyl palmitate $25000 \mathrm{IU} /$ day

- Endpoint: designed for 110000 person-years to detect a $23 \%$ reduction in lung cancer incidence

- Secondary endpoints: total and cardiovascular mortality

- Many ancillary studies/publications

ATBC, alpha-tocopherol (vitamin E), beta-carotene trial; CARET, $\beta$-carotene and retinol efficacy trial; $\mathrm{NCl}$, National Cancer Institute.

together with other chemoprevention trials, in Table 3. Cardiovascular mortality was also increased, despite the beguiling fact that $\beta$-carotene is carried in the lipoprotein particles in the circulation and was presumed to be wellpositioned to protect lipoproteins from oxidation in the circulation and, perhaps, in atherogenic lesions in the arterial walls.

CARET's study design (Thornquist et al., 1993) included 'stopping rules' in case of striking favorable or unfavorable results before the scheduled completion of the intervention, with active oversight from an independent external Safety and Endpoints Monitoring Committee. The agreed-upon O'Brien-Fleming monitoring boundaries were $0.0006,0.015$, and 0.047 for $P$-values at the first interim, second interim, and final scheduled analyses (Bowen et al., 2003). ATBC reported its adverse effects of $\beta$-carotene on April 14, 1994 (ATBC, 1994); National Cancer Institute (NCI) had notified principal investigators (PIs) of other studies on 25 March. We informed our Safety and Endpoints Monitoring Committee and PIs immediately, and prepared a letter to each of the 18314 participants (or next-of-kin), which was mailed 3 days before the announcement of the ATBC results and generally reached our participants (not 'subjects') a day before or on the day of the announcement. The media coverage was rather calm, reflecting disbelief in the results. For example, the New York headline was 'Vitamin Supplements Are Seen as No Guard against Diseases: Study upsets View Concerning Heart and Cancer'. The Seattle Post-Intelligencer declared 'Beta Carotene's Benefit Disputed'. Some smaller papers captured more of the content of the article in their headlines. The Abilene (Texas) Reporter-News wrote, 'Completely Unexpected: Major Study Suggests Beta Carotene Causes Cancer'. The Seattle Times wrote, 'Beta Carotene May
Cause, Not Prevent, Cancer'. Most epidemiologists put such emphasis on observational associations that they too reacted with disbelief.

Within CARET, the first interim analysis in August 1994 found excess cases in the active intervention arm, but the differences were not statistically significant. In September 1995, however, an accelerated second analysis found higher lung cancer incidence $(P=0.053)$, higher all-cause mortality $(P=0.014)$, and very low conditional probability of reversing the findings to show a statistically significant benefit by the scheduled end of the follow-up with 110000 person-years of treatment and observation. Extensive audits of the data set, continued data collection and endpoint determination, and special external reviews continued for 3 months, leading to a recommendation to the PI that termination of the active intervention should be considered. The combined evidence of ATBC and CARET made it hard to justify strict adherence to the guidance of the O'Brien-Fleming boundaries. Only at this point was the PI permitted to share the emerging CARET results with his colleagues on the Steering Committee, representing the study centers and the coordinating center, by conference call on January 5, 1996. It was a shock to all. Six days later, all participated in an emergency meeting, agreed that the trial's active intervention must be terminated, commissioned a letter to all the participants, and discussed the presentation of results. A manuscript was completed and submitted to a well-prepared New England Journal of Medicine, which waived its restrictions on notification of the public, so that a press conference could be held on January 18 at the National Cancer Institute in Bethesda, Maryland, together with regional press conferences at the six CARET study centers in Seattle, Washington; Portland, Oregon; San Francisco, California; Irvine, California; Baltimore, Maryland; and New London, Connecticut.

Again our letter to each participant was sent in time to be received by the vast majority before or on the day of these announcements, which drew very extensive media coverage. We established a phone bank to be ready for calls from participants, families, and media - potentially angry or anguished calls. In fact, we had only very small numbers of calls at the coordinating center and at the study centers; many of the calls were to inquire about the consequences for the staff with whom our participants had become well-acquainted (Bowen et al., 2003).

Postintervention follow-up was given a high priority by the investigators and the NCI. Follow-up results for ATBC (Virtamo et al., 2003; Tornwall et al., 2004) and for CARET (Goodman et al., 2004) were published in 2003 and 2004. ATBC found no statistically significant further increase in lung cancer [relative risk (RR) 1.6; confidence interval (CI) $0.94,1.20]$, but a continued slight excess 
total mortality (RR 1.07; CI 1.02, 1.12), attributed to cardiovascular disease during the first 4-6 years after the trial. Six-year follow-up in CARET participants showed a lung cancer RR of 1.12 (CI 0.97, 1.31) and a total mortality RR of 1.08 (CI 0.99, 1.17), suggesting persistent adverse effects but no longer statistically significant. Surprisingly, the excess in total, lung cancer, and cardiovascular mortality was largely attributable to the female participants.

\section{Lessons from $\beta$-carotene and retinol efficacy trial for potential crisis management in trials}

Many valuable lessons were obtained from CARET for the organization, conduct, and completion of large-scale prevention trials. First, unexpected and unwanted events may arise in a well-planned, long-term trial. A wellcoordinated study with up-to-date documentation, communication channels, and lines of decision-making can respond in a crisis. Early closure of an intervention has major implications for all concerned - the participants, the staff, and investigators elsewhere.

The CARET participants - who came to view themselves through personal interactions with stable study center staff and our newsletters and communications as our 'partners in the research' - reacted in an amazingly positive and calm fashion. The staff reactions, in contrast, were more negative, including guilt, anxiety, and anger, both about the results of the trial and their own employment risks. We realized that there were few precedents, especially with prevention trials, so we felt it important to share our experiences at national meetings, through publication, and in training programs. This paper continues that effort.

At the scientific level, it is essential to recognize that vitamins and pharmaceuticals are chemicals that may and do have many more actions than are well-known at the start of even the best-prepared trial. Perhaps the analogy of looking for one's lost keys under a lamp post even though the keys were lost elsewhere fits the preoccupation with $\beta$-carotene as an antioxidant. It turns out, as was discovered and reported during the many years of this long-term trial, that $\beta$-carotene has many other actions (see Omenn, 1998; Salgo et al., 1999).

Among many ancillary publications, we have also shared our experience in streamlining institutional review board (IRB) reviews for multisite trials (Thornquist et al., 2002). Just from 1988 to 1996, CARET had 441 IRB submissions for grant proposals, protocol modifications, ancillary studies, and annual reviews across the nine institutions responsible for IRB reviews. From 1989, the coordinating center drafted standardized IRB applications and informed consent documents, which were sent to the study center PIs and IRBs. We were able to guarantee consistent applications; nevertheless, the IRB responses were inconsistent. The most dramatic one was related to ancillary studies involving genotyping for potential polymorphic genes that might influence the development of particular cancers or response to various agents. In 1996, CARET, with encouragement from NCI, developed a single-study IRB cooperative agreement and negotiated its acceptance with the national office for protection of research participants. It took 2.5-4 years to complete individual cooperative agreements across our nine institutions for the six study center sites. Once in place, this mechanism reduced the average time to complete IRB approval from 6 months to 1 month for each of many substudies.

\section{Interpretations and explanations of the results (Table 4)}

The primary results of CARET can be interpreted in two stages. First was the lack of benefit. This result is shared by every other trial of $\beta$-carotene in developed countries with every endpoint. As with observational epidemiologic studies, both CARET and ATBC found a striking inverse relationship within the study populations between baseline estimates of dietary intake of fruits and vegetables, or $\beta$-carotene, or measured levels of $\beta$-carotene, and subsequent incidence rates for lung cancers. Thus, the finding of no benefit could be due to too low dosage, or too limited duration, or too late initiation of the intervention after decades of exposure to the responsible carcinogen(s). Perhaps the specific agent $\beta$-carotene (or $\beta$-carotene in combination with vitamin A or vitamin $\mathrm{E}$ ) is not effective, while other components of fruits and vegetables, even other carotenoids or retinoids, might be effective. Or $\beta$-carotene and even fruits and vegetables may be a covariable with some other protective agent. Perhaps no single agent can be as beneficial as a complex mixture from foods (see Box). Maybe there is a benefit, but it is limited to a subgroup. In fact, we observed a favorable point estimate of RR for the 6000 former smokers (RR 0.80 with CI $0.48,1.31 ; P=0.37$ ), which was a very tempting finding that might reinforce the value of stopping smoking and staying off the habit. The confidence interval, however, considerably overlapped that of the current smoker subpopulation, and the former heavy smokers, stunningly, had even higher incidence of increased cardiovascular mortality (Omenn et al., 1996a, b).

\footnotetext{
'Take any new reports of disease-preventing vitamins with a grain of salt. When this is all sorted out, it could turn out that we will all have to take 50 or 60 different nutrients to get a real benefit. 'That's a possibility', says Goodman, of the Hutchinson Cancer Research Center. 'It's called broccoli.'

P.E. Ross, Spinach in a pill. Forbes, November 4, 1996; 354-355.
}

The second finding, besides no benefit, is serious adverse effects. Perhaps the dosage or cumulative dose was too 
high, leading to toxicity only at high levels. Both CARET and ATBC, however, examined results by tertile of serum $\beta$-carotene concentration after reaching a stable treatment level, and found no evidence of concentration of lung cancers or deaths in the highest $\beta$-carotene group. There might be especially susceptible subgroups, like current smokers, or less susceptible subgroups, like never smokers, who were basically unrepresented in these trials. Never smokers, of course, have very low risk of lung cancers from which a reduction would be essentially impossible to demonstrate. Perhaps the synthetic $\beta$-carotene in supplements is chemically different from 'natural' $\beta$-carotene in foods or in alga-derived supplements. Clear evidence exists that the chemistry is identical, and the proportions of 9-cis, 13-cis, and all-trans isomers, which do differ, rapidly become interconverted to similar proportions once ingested and circulated in the body (Omenn, 1998). Finally, there may be toxic mechanisms not previously recognized. This interpretation is almost surely correct. The trial results stimulated a whole new generation of laboratory work, which has shown oxidation of the alternating double bonds in $\beta$-carotene, which may be particularly salient in the lungs and the arterial circulation. These derivatives produce increased benzo $[a]$ pyrene-DNA adducts in experimental systems (Thornquist et al., 2002). Beta-carotene together with cigarette smoke increased key steps in carcinogenic pathways, like c-jun, c-fos, and AP-1 in the ferret, which absorbs and metabolizes $\beta$-carotene more like humans than do the rodents (Wang et al., 1999). The study did indicate that low doses might be associated with lower risk. Subsequently, a study of $\beta$-carotene and lutein in azoxymethane-induced colon carcinogenesis, using a surrogate endpoint of aberrant cryptic foci, found reduced foci at low doses and increased foci at high doses of each agent (Raju et al., 2005).

Additional reports of adverse effects in clinical trials have appeared (Bairati et al., 2005). For example, in a trial with $\alpha$-tocopherol to prevent second primaries in patients surviving head and neck cancers, Bairati et al. (2005) found much elevated incidence of second primaries (HR 2.88, 95th CI 1.56, 5.31) during the supplementation period, although lower rates were found upon discontinuation of the regimen (median 52 months).

Table 4 Relative risks from studies

\begin{tabular}{lccc}
\hline & $N$ & Lung cancer & Mortality \\
\hline ATBC & 874 & $1.18(1.03-1.36)$ & $1.08(1.01-1.16)$ \\
CARET & 388 & $1.28(1.04-1.57)$ & $1.17(1.03-1.33)$ \\
PHS & 170 & $0.93(0.69-1.26)$ & $1.02(0.93-1.11)$ \\
Tyler & & $1.24(0.78-1.96)$ & - \\
Linxian & 31 & $0.55(0.26-1.14)$ & $0.91(0.84-0.99)$ \\
\hline
\end{tabular}

ATBC, alpha-tocopherol, beta-carotene trial; CARET, $\beta$-carotene and retinol efficacy trial; PHS, Physicians' health study.

\section{Future for lung cancer chemoprevention (Table 5)}

Despite these disappointments, we should be optimistic about chemoprevention of lung cancers. Many more candidate agents exist in the drug development pipeline and many new targets in signaling pathways, including targets being explored for therapeutic agents (Willis and Colburn, 2002; Steele et al., 2003; Subbaramaiah and Dannenberg, 2003; Omenn, 2004a, b; Hirsch and Lippman, 2005; Kim and Hong, 2005). There is also interest in randomized trials of foods, although initial results, for example, using high-fiber cereals to prevent colon polyp recurrence, have not been encouraging (Alberts et al., 2000).

First, there is a sustained interest in retinoids. These synthetic relatives of vitamin A play a major role in regulating differentiation, growth, and apoptosis of many cell types. They have known nuclear receptors (RAR/ RXR), with admittedly complex biology. There are multiple retinoids and rexinoids to test. Most experience to date in humans has been with 4-hydroxy-retinamide. As we noted many actions of $\beta$-carotene, there is evidence of multiple sites of action of retinoids outside the RAR/ RXR receptors - on c-Myc, Ap-1, cell surface death receptors, reactive oxygen, mitochondrial permeability, 12-lipoxygenase, nuclear factor- $\kappa \mathrm{B}$, ubiquitination, cyclooxygenase-2 (Cox-2), and tumor necrosis factor $\alpha-$ for starters. Unfortunately, there have been few new clinical trials reports in recent years (Hirsch and Lippman, 2005; Kim and Hong, 2005).

Other molecular targets for chemoprevention exist. These include genes with altered expression or activity causally related to carcinogenesis, some with known inhibitors, such as nonsteroidal anti-inflammatory drugs and celecoxib and rofecoxib for Cox-2; Raf kinase inhibitor for ras mutations/Raf kinase; KP-SD-1, (for Kinetek Pharmaceuticals SD-1, Kinetek Pharmaceuticals, Vancouver, British Columbia, Canada), inhibitor of integrin-linked kinase; and metalloprotease inhibitors for matrilysin (MMP-7). Other genes/targets with causal relationships, such as $\mathrm{p} 27$, protein kinase $\mathrm{C}$ delta, and

\section{Table 5 Strategic conclusions for chemoprevention of lung} cancers

- Lung cancers are highly heterogeneous

- Many targets are in other cells than the tumor cells themselves-inflammatory, endothelial, stromal

- Validated molecular markers and surrogate endpoints are essential

- Suspect all agents have multiple effects (adverse)

- Therapeutic agents have major advantages for chemopreventive drug development

- Test agents for efficacy against other cancers/other diseases, especially with a possibly shared mechanism ( $>1$ endpoint/trial)

- Investment in chemoprevention, starting with research, can be justified by the potential impact 
AP-1, have no publicly known drugs yet. For some targets, drugs are available, but the causal significance is uncertain; an example is octreonide acetate for insulinlike growth factor. Finally, there are both known and uncertain targets for such dietary components as monoterpenes, isothiocyanates (P450 enzymes), myo-inositol (inositol hexaphosphate), vitamins $\mathrm{E}$ and $\mathrm{C}$, selenium, isoflavones, curcumin in curry, phytosterols in soybeans, polyphenols in green tea, and resveratrol in berries.

Before the increasing evidence of adverse cardiovascular effects from Cox-2 inhibitors, these agents were particularly promising, owing to their Food and Drug Administration-approved status, the evidence of reduction in recurrence rate of colon polyps in patients with familial polyposis, and their potentially chemopreventive actions (Steele et al., 2003; Subbaramaiah and Dannenberg, 2003). These actions include antiangiogenesis, antiproliferation, antioxidation, apoptosis, and immune system effects.

A final category of promising agents comprises lipoxygenase inhibitors, whose actions include antiangiogenesis, antiproliferation, antioxidation, apoptosis induction, and anti-inflammatory responses to various 12-lipoxygenase and 5-lipoxygenase agents (Steele et al., 2003; Subbaramaiah and Dannenberg, 2003).

\section{Acknowledgements}

The findings and conclusions presented in this paper were presented in large part at the Translational Cancer Prevention Workshop in Haifa, Israel, 4-6 May, 2004, and the International Educational Conference on Cancer Prevention at the Dead Sea, Israel, 8 May, 2004, both chaired by Gad Rennert, MD.

I thank my co-investigators in CARET: Gary Goodman, Mark Thornquist, Matt Barnett, Cim Edelstein, Scott Barnhart, Linda Rosenstock, Drew Brodkin, Deborah Bowen, and Sam Hammar (Seattle); James Keogh (Baltimore), Frank Meyskens Jr and James Williams Jr (Irvine), Mark Cullen and Martin Cherniack (Connecticut), Barbara Valanis and Andrew Glass (Portland), and John Balmes, Jim Cone, and Diane Liu (San Francisco) at these additional study centers; our excellent staff; and our NCI program officer Marjorie Perloff. Most importantly, we all thank our 'partners in the research', the volunteer participants in CARET and their families.

\section{References}

Alberts DS, Martinez ME, Roe DJ, Guillen-Rodriguez JM, Marshall JR, van Leeuwen JB, et al. (2000). Lack of effect of a high-fiber cereal supplement on the recurrence of colorectal adenomas. N Engl J Med 342:1156-1162.

Alpha-Tocopherol, Beta-Carotene Cancer Prevention Study Group (ATBC) (1994). The effect of vitamin $E$ and beta-carotene on the incidence of lung cancer and other cancers in male smokers. N Engl J Med 330:1029-1035.
Bairati I, Meyer F, Gelinas M, Fortin A, Nabid A, Brochet F, et al. (2005). A randomized trial of antioxidant vitamins to prevent second primary cancers in head and neck cancer patients. J Natl Cancer Inst 97:481-486.

Beer DG, Kardia SLR, Huang C-C, Ghafoor A, Samuels A, Ward E, et al. (2002). Gene-expression profiles predict survival of patients with lung adenocarcinoma. Nat Med 8:816-824.

Bowen DJ, Thornquist M, Anderson K, Barnett M, Powell C, Goodman G, Omenn GS (2003). Stopping the active intervention: CARET. Controlled Clin Trials 24:39-50.

Freedman LS, Barchana M, Al-Kayed S, Qasem MB, Young JL, Edwards BK, et al. (2003). A comparison of population-based cancer incidence rates in Israel, and Jordan. Eur J Cancer Prev 12:359-365.

Goodman GE, Thornquist MD, Balmes J, Cullen MR, Meyskens FL Jr, Omenn GS et al. (2004). The beta-carotene and retinol efficacy trial: incidence of lung cancer and cardiovascular disease mortality during 6-year follow-up after stopping beta-carotene and retinol supplements. J Natl Cancer Inst 96:1743-1750.

Greenwald P (2002). Cancer prevention clinical trials. J Clin Oncol 20:14s-22s.

Hirsch FR, Lippman SM (2005). Advances in the biology of lung cancer chemoprevention. J Clin Oncol 23:3186-3197.

IARC Monographs on the Evaluation of Carcinogenic Risks to Humans (2004). Tobacco smoke and involuntary smoking. Vol. 83. Lyon, France: WHO/ International Agency for Research on Cancer.1452pp.

Jemal A, Tiwari RC, Murray T, Ghafoor A, Samuels A, Ward E, et al. (2004). Cancer statistics, 2004. CA Cancer J Clin 54:8-29.

Keith RL, Miller YE (2005). Lung cancer: genetics of risk and advances in chemoprevention. Curr Opin Pulm Med 11:265-271.

Kim ES, Hong WK (2005). An apple a day "..." does it really keep the doctor away? The current state of cancer chemoprevention. J Natl Cancer Inst 97:468-470.

McCormick DL, Burns FJ, Albert RE (1980). Inhibition of rat mammary carcinogenesis by short dietary exposure to retinyl acetate. Cancer Res 40:1140-1143.

Omenn GS (1998). Chemoprevention of lung cancer: the rise and demise of beta carotene. Ann Rev Public Health 19:73-99.

Omenn GS (2004a). Cancer prevention, chapter 190. In: Goldman L, Ausiello D, editors. Cecil textbook of medicine. 22 ed. Philadelphia, PA: W.B. Saunders. pp. 1134-1137.

Omenn GS (2004b). Human lung cancer chemoprevention strategies: Parker B. Francis Lecture. Chest 125:123S-127S.

Omenn GS, Goodman GE, Thornquist MD, Balmes J, Cullen MR, Glass A, et al (1996a). Effects of a combination of $\beta$-carotene and vitamin $A$ on lung cancer and cardiovascular disease. N Engl J Med 334:1150-1155.

Omenn GS, Goodman GE, Thornquist MD, Balmes J, Cullen MR, Glass A, et al. (1996b). Risk factors for lung cancer and for intervention effects in CARET, the beta-carotene and retinol efficacy trial. J Natl Cancer Inst 88:1550-1559.

Peto R, Doll R, Buckley JD, Sporn MB (1981). Can dietary beta-carotene materially reduce human cancer rates? Nature 290:201-208.

Raju J, Swamy MV, Cooma I, Patlolla JMR, Pittman B, Reddy BS, et al. (2005). Low doses of beta-carotene and lutein inhibit $A O M$-induced rat colonic ACF formation but high doses augment ACF incidence. Int $J$ Cancer 113:798-802.

Saffiotti U, Montesano R, Sellakumar AR, Borg SA (1967). Experimental cancer of the lung. Inhibition by vitamin $A$ of the induction of tracheo-bronchial squamous metaplasia and squamous cell tumors. Cancer 20:857-864.

Salgo MG, Cueto R, Winston GW, Pryor W (1999). Beta carotene and its oxidation products have different effects on microsome mediated binding of benzo[a]pyrene to DNA. Free Radic Biol Med 26:162-173.

Sporn MB, Dunlop NM, Newton DL, Smith JM. (1976). Prevention of chemical carcinogenesis by vitamin $\mathrm{A}$ and its synthetic analogs [retinoids]. Fed Proc 35:1332-1338.

Steele VE, Hawk ET, Viner JL, Lubet RA (2003). Mechanisms and applications of non-steroidal anti-inflammatory drugs in the chemoprevention of cancer. Mutat Res 523:137-144.

Subbaramaiah K, Dannenberg AJ (2003). Cyclooxygenase 2: a molecular target for cancer prevention and treatment. Trends Pharmacol Sci 24:96-102.

Thornquist M, Omenn GS, Goodman GE, Grizzle J, Rosenstock L, Barnhart S, et al. (1993). Statistical design of the carotene and retinol efficacy trial (CARET). Controlled Clin Trials 14:308-324.

Thornquist MD, Edelstein C, Goodman GE, Omenn GS (2002). Streamlining IRB review in multisite trials through single-study IRB Cooperative Agreements: experience of the $\beta$-Carotene and Retinol Efficacy Trial (CARET). Controlled Clin Trials 23:80-86.

Tornwall ME, Virtamo J, Korhonen PA, Virtanen MJ, Taylor PR, Albanes D, et al. (2004). Effect of alpha-tocopherol and beta-carotene supplementation on 
coronary heart disease during the 6-year post-trial follow-up in the ATBC study. Eur Heart J 25:1171-1178.

Virtamo J, Pietinen P, Huttunen JK, Korhonen P, Malila N, Virtanen MJ, et al. (ATBC Study Group) (2003). Incidence of cancer and mortality following alphatocopherol and beta-carotene supplementation: a post-intervention follow-up. JAMA 290:476-485.
Wang XD, Liu C, Bronson RT, Smith DE, Krinsky NI, Russell M (1999). Retinoid signaling and activator protein-1 expression in ferrets given beta-carotene supplements and exposed to tobacco smoke. J Natl Cancer Inst 91:60-66.

Willis DB, Colburn NH (2002). Molecular targets for cancer prevention: a meeting review of the third American Cancer Society Schilling Research Conference. Cancer Epidemiol Biomarkers Prev 11:972-978. 